Pacific Journal of Mathematics

FIELDS OF AUTOMORPHISMS AND DERIVATIONS O Januario VARELa 


\title{
FIELDS OF AUTOMORPHISMS AND DERIVATIONS OF $C^{*}$-ALGEBRAS
}

\author{
JANUARIO VARELA
}

Let $A$ be a $C^{*}$-algebra with identity; the group of star automorphisms of $A$ leaving its center elementwise fixed is represented as the set of all global sections of a field of groups whose base space is the spectrum of the center of $A$ and whose stalks are groups of automorphisms of a family of quotient $C^{*}$-algebras. The derivations of $A$ are similarly represented in a field of Lie algebras.

Introduction. The representation of $C^{*}$-algebras as sections in bundles was used by Tomiyama and Takesaki [7] and others to study the automorphisms of these algebras. On the other hand, Teleman [6] applied representations of $W^{*}$-algebras as sections in a sheaf to obtain information about their Lie algebra of derivations. For biregular rings a description of the group of automorphisms has also been achieved by Dauns and Hofmann in sheaf theoretical terms [1]. An analytic version of some of these results is presented here via the representation of a $C^{*}$-algebra as sections in a uniform field.

Given a $C^{*}$-algebra $A$ with identity, there is a bundle or sheaflike structure called field and denoted by $(E, \pi, T)$, whose ingredients are: The maximal ideal space $T$ of the center of $A$ equipped with the hull-kernel topology and a disjoint union $E$ of a family of quotient $C^{*}$-algebras $\left\{A / I_{t} \mid t \in T\right\}$ topologized in such a way that the projection $\pi: E \rightarrow T$ and the cross sections $\hat{a}: t \mapsto a+I_{t}: T \rightarrow E$ are continuous for every $a \in A$. The existence of such a topology depends on the upper semicontinuity of the functions $t \mapsto\left\|a+I_{t}\right\|$ : $T \rightarrow \boldsymbol{R}_{+}$. If the primitive ideal space $\operatorname{Prim} A$ is Hausdorff, $T$ is homeomorphic to $\operatorname{Prim} A$ and for the stalk above $P \in \operatorname{Prim} A$, one can take the simple algebra $A / P$. More generally, $T$ is the StoneČech compactification of Prim $A$ and $I_{t}$ is the closed two sided ideal generated by $t$. In [3] 4.7 Halpern showed that if $A$ is a $W^{*}$ algebra, $I_{t}$ is a primitive ideal of $A$ for every $t \in T$, and that $\left\{I_{t} \mid t \in T\right\}$ (which is homeomorphic to $T$ ) is dense in Prim $A$. The set of all continuous bounded global sections of this field is denoted by $\Gamma(\pi)$, and is naturally endowed with a $C^{*}$-algebra structure with respect to point-wise defined operations and the norm given by $\|\sigma\|=\{\|\sigma(t)\| \mid t \in T\}$. The non commutative version of the Gelfand Naimark theorem ([2], Chap. III, 8.14) says that $A$ is isometrically star isomorphic to $\Gamma(\pi)$. One can also decompose $A$ over the spectrum 
of any closed subalgebra of the center of $A$ containing the identity [8].

The duality between $C^{*}$-algebras and fields of $C^{*}$-algebras [8] indicates that all the information relative to the group of automorphisms of a $C^{*}$-algebra $A$ can be found in the canonical field $(E, \pi, T)$ associated to $A$.

A description of the group $N$ of star automorphisms of $A$ leaving the center elementwise fixed is given in terms of the groups of automorphisms of the fibers of $(E, \pi, T)$; to this effect a field of groups is constructed, and $N$ is realized as the group of all global sections. The derivations of $A$ are similarly described in terms of the derivations of the stalks of the field $(E, \pi, T)$. This paper is part of the author's dissertation at Tulane University. I would like to take this opportunity of expressing my sincere gratitude to professors J. Dauns and K. H. Hofmann my research supervisors, for their advice and encouragemént.

1. Terminology. For the foundation of the theory of sectional representation we refer to $[2,4]$. A few definitions follow to establish notation and terminology.

Definition 1.1. Let $E$ and $T$ be topological spaces and $\pi: E \rightarrow T$ a surjective continuous map, then $(E, \pi, T)$ is called a field or bundle of topological spaces, $T$ is the base space and $\pi^{-1}(t)$ the stalk or fiber above $t$.

DEFinition 1.2. A local selection is a map $\sigma: Q \rightarrow E$ whose domain $Q$ is an open subset of $T$ and $\pi(\sigma(t))=t$ for every $t \in Q$. If $Q=T$, then $\sigma$ is a global selection. A continuous selection is called section. The set of all global sections is denoted by $\Gamma(\pi)$.

Definition 1.3. Let $(E, \pi, T)$ be a field of topological spaces and $\mathscr{U}$ a filter on $E \vee E=\{(x, y) \in E \times E \mid \pi(x)=\pi(y)\}$ such that the filter [थ] generated by $\mathscr{C}$ on $E \times E$ is a uniform structure. $(E, \pi, T)$ is a uniform field if the neighborhood filter of every point $x \in E$ has a basis given by the sets $U(\sigma)=\{y \in E \mid \pi(y) \in \operatorname{dom} \sigma$ and $(\sigma(\pi(y)), y) \in U\}$ where $\sigma$ is a local section such that $\sigma(\pi(x))=x$, dom $\sigma$ is the domain of $\sigma$ and $U$ is an element of the uniformity $\mathscr{U}$.

DEFINITION 1.4. Let $(E, \pi, T)$ be a uniform field whose stalks are normed spaces and whose uniformity is given by the fundamental entourages $U=\{(x, y) \in E \vee E \mid\|x-y\|<\varepsilon\}$ with $\varepsilon>0$, then $(E$, $\pi, T)$ is a field or bundle of normed spaces if the following functions 
are continuous:

$$
(x, y) \longmapsto x+y: E \vee E \longrightarrow E \text { and }(\alpha, x) \longmapsto \alpha x: C \times E \longrightarrow E \text {. }
$$

If the stalks of a field of normed spaces are $C^{*}$-algebras and if the functions $(x, y) \mapsto x y: E \vee E \rightarrow E$ and $x \mapsto x^{*}: E \rightarrow E$ are continuous, then $(E, \pi, T)$ is a field or bundle of $C^{*}$-algebras. A field of $C^{*}$ algebras such that for every $t \in T$ the fiber $E_{t}$ above $t$ has an identity $\zeta(t)$ is a field of $C^{*}$-algebras with identity provided the selection $t \mapsto \zeta(t): T \rightarrow E$ is continuous. The set of all bounded global sections of $(E, \pi, T)$ is then a $C^{*}$-algebra with respect to pointwise defined operations, by taking the sup-norm $\|\sigma\|=\sup \{\|\sigma(t)\| \mid t \in T\}$ and is denoted by $\Gamma^{b}(\pi)$.

DEFINITION 1.5. A set $\Sigma$ of local (resp. global) selections of a field of topological spaces is called full, if for every $x \in E$ there exists $\tau \in \Sigma$ such that $\tau(\pi(x))=x$.

Definition 1.6. Let $\sigma$ be a local section in a field of normed spaces $(E, \pi, T)$, then $\{x \in E \mid \pi(x) \in \operatorname{dom} \sigma$ and $\|x-\sigma(\pi(x))\|<\varepsilon\}$ is called the $\varepsilon$-tube around $\sigma$ and is denoted by $\mathscr{T}(\sigma, \varepsilon)$.

LEMMA 1.7. Let $(E, \pi, T)$ be a field of normed spaces. The following two statements are equivalent:

(1) For every local section $\sigma$, the function $t \mapsto\|\sigma(t)\|: \operatorname{dom} \sigma \rightarrow$ $\boldsymbol{R}_{+}$is upper semicontinuous.

(2) For every local section $\sigma$ and every $\varepsilon>0$, the $\varepsilon$-tube around $\sigma, \mathscr{T}(\sigma, \varepsilon)$ is an open set in $E$.

Proof. If the function $t \mapsto\|\sigma(t)\|$ is upper semicontinuous, then for every $\varepsilon>0$, the set $\{t \in \operatorname{dom} \sigma \mid\|\sigma(t)\|<\varepsilon\}$ is open in $T$. Let $x \in E$ such that $\|\sigma(\pi(x))-x\|<\varepsilon$, then there is a $\delta, 0<\delta<\varepsilon$ such that $\|\sigma(\pi(x))-x\|<\varepsilon-\delta$. If $\tau$ is a local section for which $\tau(\pi(x))=x$, the $Q=\{t \in T \mid\|\sigma(t)-\tau(t)\|<\varepsilon-\delta\}$ is an open neighborhood of $\pi(x)$. The restriction $\tau_{Q}$ of $\tau$ to $Q$ is then a local section such that $\tau_{Q}(\pi(x))=x$ and $\operatorname{Im} \tau_{Q}$ is contained in $\mathscr{T}(\sigma, \varepsilon-\delta)$. Therefore $\mathscr{T}\left(\tau_{Q}, \delta\right)$ is a neighborhood of $x$ and $\mathscr{T}\left(\tau_{Q}, \delta\right) \subset \mathscr{T}(\sigma, \varepsilon)$. Then $\mathscr{T}(\sigma, \varepsilon)$ is open in $E$.

The converse follows from [2], Chapter I, 1.30 .

REMARK 1.8. If $T$ is assumed to be uniformizable, the above lemma can be formulated in term of global sections, since the existence of a full set of local sections implies the existence of a full set of global sections, indeed if $\tau$ is a local section through 
$x \in E$ and $f: T \rightarrow[0,1]$ is continuous function such that $f(\pi(x))=1$ and $f(s)=0$ for $s \in T \backslash \operatorname{dom} \sigma$, then $\sigma$ defined by $\sigma(s)=f(s) \tau(s)$ if $s \in \operatorname{dom} \tau$ and $\sigma(s)=0$ if $s \in T \backslash \operatorname{dom} \tau$, is a global section through $x$.

REMARK 1.9. Let $(E, \pi, T)$ be a field of normed spaces over a quasicompact space $T$. If the function $t \mapsto\|\sigma(t)\|: T \rightarrow \boldsymbol{R}_{+}$is upper semicontinuous for every global section $\sigma$, then $\sigma$ is bounded.

Proof. The semicontinuity of $t \mapsto\|\sigma(t)\|$ for $\sigma \in \Gamma(\pi)$ implies that the sets $\{t \in T\|\| \sigma(t) \|<n\}, n=1,2, \ldots$ form an open covering of $T$ and since $T$ is quasicompact, then $\sigma$ is bounded.

Convention 1.10. From now on we will assume that $t \mapsto\|\sigma(t)\|$ is upper semicontinuous for every global section in a field $(E, \pi, T)$ of normed spaces, unless otherwise specified.

\section{Fields of linear maps.}

2.1 Let $(E, \pi, T)$ be a field of normed spaces. Denote by $\mathscr{B}_{t}$ the algebra of all bounded linear transformations of $E_{t}=\pi^{-1}(t)$. Let $\mathscr{D}_{0}$ be the disjoint union of the family $\left\{\mathscr{B}_{t} \mid t \in T\right\}$ and $\rho: \mathscr{D}_{0} \rightarrow T$ defined by $\rho(u)=t$ if $u \in \mathscr{B}_{t}$. Let $\Sigma$ be a normed space consisting of a full set of bounded global sections of $(E, \pi, T)$.

Our aim is to define a uniform field $(\mathscr{D}, \rho, T)$ over the same topological space $T$, with $\mathscr{D} \subset \mathscr{D}_{0}$ appropriately chosen. Later in $\S 3$, we will consider a subfield of $(\mathscr{D}, \rho, T)$ whose stalks are groups of isometries, and in $\S 6$ another subfield whose stalks are Lie algebras of derivations will be studied.

2.2. We endow $\mathscr{D}_{0}$ with the coarsest topology making continuous the functions

$$
\begin{aligned}
& u \longmapsto \rho(u): \mathscr{D}_{0} \longrightarrow T \text { and } \\
& u \longmapsto u(\sigma(\rho(u))): \mathscr{D}_{0} \longrightarrow E \text { for every } \sigma \in \Sigma .
\end{aligned}
$$

2.3. Let $u$ be an arbitrary element of $\mathscr{D}_{0}$, denote by $Q$ an open neighborhood of $t=\rho(u)$, choose sections $\sigma$ and $\tau$ in $\Sigma$ such that $u \sigma(t)=\tau(t)$ and take $\varepsilon>0$.

Define $M(u)=M(u, \sigma, \tau, Q, \varepsilon)$ as follows

$$
M(u)=\left\{v \in \mathscr{D}_{0} \mid \rho(v) \in Q \text { and } v(\sigma(\rho(v))) \in \mathscr{T}(\tau, \varepsilon)\right\} .
$$

Then the filter of neighborhoods of $u$ is generated by the sets of the form $M(u)$. 
2.4. For a local selection $\lambda$ in $(\mathscr{D}, \rho, T)$ the following two statements are equivalent:

(1) $t \mapsto \lambda(t): \operatorname{dom} \lambda \rightarrow \mathscr{D}_{0}$ is a local section.

(2) $t \mapsto \lambda(t) \sigma(t): \operatorname{dom} \lambda \rightarrow E$ is a local section in the field $(E, \pi, T)$ for every $\sigma \in \Sigma$.

2.5. Let $\mathscr{D}$ be the subset of $\mathscr{D}$ consisting of the points through which there is a global section. Let $\sigma$ be an element of $\Sigma$ and $\varepsilon>0$.

Set $\mathscr{D} \vee \mathscr{D}=\{(u, v) \in \mathscr{D} \times \mathscr{D} \mid \rho(u)=\rho(v)\}$ and define $U(\sigma, \varepsilon)$ as follows: $U(\sigma, \varepsilon)=\{(u, v) \in \mathscr{D} \vee \mathscr{D} \mid\|u \sigma(\rho(u))-v \sigma(\rho(v))\|<\varepsilon\}$. It is immediate that the finite intersections of sets of the form $U(\sigma, \varepsilon)$ are a fundamental system of entourages of a uniformity $\mathscr{U}$ for $\rho$.

REMARK. One should observe, that $\mathscr{D}$ may consist of the identity section only

2.6. The uniformity $\mathscr{\mathscr { C }}$ for $\rho$ induces the topology defined in $\mathscr{D}$ and hence $(\mathscr{D}, \rho, T)$ is a uniform field.

Proof. Let $u$ be an element of $\mathscr{D}$ and $\xi$ a global section in $(\mathscr{D}, \rho, T)$ such that $\xi(\rho(u))=u$, denote by $\xi_{Q}$ the restriction of $\xi$ to an open neighborhood $Q$ of $\rho(u)$, then

$$
U(\sigma, \varepsilon)\left(\xi_{Q}\right)=\{v \in \mathscr{D} \mid \rho(v) \in Q \text { and }\|\xi(\rho(v)) \sigma(\rho(v))-v \sigma(\rho(v))\|<\varepsilon\}
$$

is a neighborhood of $u$ in $\mathscr{D}$ because by 2.4

$$
t \longmapsto \xi(t) \sigma(t): T \longmapsto E \text { is a global section in }(E, \pi, T) \text {. }
$$

Conversely, given a neighborhood of $u$ of the form $M(u, \sigma, \tau, Q, \varepsilon)$, let $\lambda$ be a global section in $(\mathscr{D}, \rho, T)$ such that $\lambda(\rho(u))=u$. If we set $t=\rho(u)$, then $u \sigma(t)=\tau(t)$ and $\lambda(t) \sigma(t)=\tau(t)$, hence there is a neighborhood $P$ of $t$ contained in $Q$ such that for every $s$ in $P$, $\|\lambda(s) \sigma(s)-\tau(s)\|<\varepsilon / 2$. If $\lambda_{P}$ is the restriction of $\lambda$ to $P$, then

$$
U(\sigma, \varepsilon / 2)\left(\lambda_{P}\right)=\{v \in \mathscr{D} \mid \rho(v) \in P \&\|\lambda(\rho(v)) \sigma(\rho(v))-v \sigma(\rho(v))\|<\varepsilon / 2\}
$$

is contained in $M(u, \sigma, \tau, Q, \varepsilon)$. Thus the topology induced by the uniformity coincides with the topology defined in $\mathscr{D}$.

\section{Field of automorphisms.}

Definition 3.1. Let $(G, p, T)$ be a uniform field such that the following conditions are satisfied.

(a) All the stalks are groups and the functions $(u, v) \mapsto u v$ : 
$G \vee G \mapsto G$ and $u \mapsto u^{-1}: G \rightarrow G$ are continuous, where $G \vee G=$ $\{(u, v) \in G \times G \mid p(u)=p(v)\}$.

(b) The uniform structure induced by the field uniformity $\mathscr{C}$ is coarser than the left (resp. right) uniform structure of the stalk induced by (a).

(c) For every entourage $U \in \mathscr{C}$ there exists $V \in \mathscr{U}$ such that

$$
V \subset\left\{(u, v) \in G \vee G \mid\left(\zeta(p(u)), u^{-1} v\right) \in U\right\}
$$

(resp. $\left.V \subset\left\{(u, v) \in G \vee G \mid\left(\zeta(p(u)), v u^{-1}\right) \in U\right\}\right)$, where $\zeta(t)$ is the unit elements of the group $G_{t}=p^{-1}(t)$.

(d) For every entourage $U \in \mathscr{C}$ and every global section $\lambda$ there exists an entourage $V \in \mathscr{C}$ such that $\left(\lambda(t) u \lambda(t)^{-1}, \lambda(t) v \lambda(t)^{-1}\right) \in U$ if $(u, v) \in V$ and $t=p(u)=p(v)$.

Then $(G, p, T)$ is called a left (resp. right) uniform field of topological groups.

The set $\Gamma(p)$ of all global sections is a group with respect to pointwise defined operations and is naturally equipped with a uniform structure $\tilde{\mathscr{C}}$ with fundamental entourages of the form $\widetilde{U}=\{(\lambda, \mu) \in \Gamma(p) \times \Gamma(p) \mid(\forall t \in T)((\lambda(t), \mu(t)) \in U)\}$ with $U \in \mathscr{U}$. The neighborhood filter of the identity $\zeta$ of $\Gamma(p)$ for the topology determined by $\tilde{\mathscr{C}}$ is given by the sets

$$
\widetilde{U}(\zeta)=\{\lambda \in \Gamma(p) \mid(\zeta, \lambda) \in \widetilde{U}\} .
$$

REMARK 3.2. Conditions (b) and (c) of Definition 3.1 imply that the field uniformity $\mathscr{U}$ of $(G, p, T)$ induces on each stalk the left (resp. right) uniform structure of the stalk induced by (a).

REMARK 3.3. The following condition implies the conditions (b) and (c) of Definition 3.1.

$\left(b^{\prime}\right)$ A fundamental system of entourages for the field uniformity $\mathscr{C}$ of $(G, p, T)$ is given by the sets of the form

$$
\left\{(u, v) \in G \vee G \mid u^{-1} v \in W(\zeta)\right\}\left(\operatorname{resp} .\left\{(u, v) \in G \vee G \mid v u^{-1} \in W(\zeta)\right\}\right)
$$

where $\zeta$ is the unit element of $\Gamma(p)$ and $W$ ranges through $\mathscr{U}$.

3.4. We recall that if $N$ is a group and $\mathscr{G}$ is a filter on $N$ satisfying the Properties I and II listed below, then there is a unique topology on $N$ with respect to which $N$ is a topological group having $\mathscr{G}$ as filter of neighborhoods of the identity.

(I) Given $U \in \mathscr{G}$ there exists $V \in \mathscr{G}$ such that $V^{-1} V \subset U$.

(II) For all $v \in N$ and $V \in \mathscr{G}$ we have $v V v^{-1} \in \mathscr{G}$. 
3.5. If $(G, p, T)$ is a left uniform field of topological groups, then $\Gamma(p)$ is a topological group.

Proof. (I) Given a neighborhood of $\zeta$ of the form $\widetilde{U}(\zeta)$ by 3.1 . (c) we can find $V \in \mathscr{U}$ such that $V \subset\left\{(u, v) \mid u^{-1} v \in U(\zeta)\right\}$.

Let $W$ a symmetric entourage such that $W \circ W \subset V$, then $\lambda \in$ $\widetilde{W}(\zeta)$ and $\mu \in \widetilde{W}(\zeta)$ imply $(\lambda, \mu) \in \widetilde{V}$ and so $\lambda^{-1} \mu \in \widetilde{U}(\zeta)$.

(II) Given $U \in \mathscr{Q}$ and $\lambda \in \Gamma(p)$ there exists $V \in \mathscr{U}$ such that $\lambda \tilde{V}(\zeta) \lambda^{-1} \subset \tilde{U}(\lambda)$ : Take the entourage $V$ corresponding to $U$ and $\lambda$ condition (d) of Definition 3.1, then $\mu \in \tilde{V}(\zeta)$ implies $\lambda \mu \lambda^{-1} \in \widetilde{U}(\zeta)$. Hence $\Gamma(p)$ is a topological group whose left uniform structure is coarser than $\tilde{\mathscr{C}}$.

ExAmple 3.6. Lt $T$ the discrete space $\boldsymbol{N} \backslash\{0\}$. Endow $\boldsymbol{Q}$ with the $p$-adic topology and $G=T \times \boldsymbol{Q}$ with the product topology. Take as uniformity $\mathscr{Q}$ for $\pi: G \rightarrow T$ the one given by the fundamental entourages

$$
U(n)=\left\{(x, y) \in G \vee G\left|h_{n}\left(\pi(x), \sup \left(|x|_{p},|y|_{p}\right)\right)\right| x-\left.y\right|_{p}<1 / n\right\}
$$

for $n=1,2, \cdots$, where $h_{n}: T \times \boldsymbol{R}_{+} \rightarrow\{0,1\}$ is the function defined by

$$
h_{n}(t, r)=\left\{\begin{array}{l}
1 \text { if } t \leqq n \\
0 \text { if } t>n \text { and } r<1+1 / n \\
1 \text { if } t>n \text { and } r \geqq 1+1 / n .
\end{array}\right.
$$

Then $(G, p, T)$ is a uniform field of topological groups, and the left uniform structure of $\Gamma(\pi)$ is strictly coarser than $\tilde{\mathscr{U}}$.

3.7. Let $A$ be an involutive normed algebra, a bijection $\lambda: A \rightarrow A$ is a star automorphism of $A$ provided $\lambda(x+y)=\lambda x+\lambda y, \lambda(x y)=$ $(\lambda x)(\lambda y), \lambda(\alpha x)=\alpha \lambda x$ and $\lambda x^{*}=(\lambda x)^{*}$ for every $x, y \in A$ and $\alpha \in C$. If $\|\lambda x\|=\|x\|$ for every $x \in A, \lambda$ is called an isometric star automorphism of $A$.

3.8. Let $(E, \pi, T)$ be a field of involutive normed algebras. Denote by Aut $E_{t}$ the group of isometric star automorphisms of $E_{t}=\pi^{-1}(t)$ and let $G_{0}$ be the disjoint union of all the groups Aut $E_{t}$. Let $\Sigma$ be an involutive normed algebra consisting of a full set of bounded global sections of $(E, \pi, T)$ and consider the uniform field $(\mathscr{D}, \rho, T)$ defined in $\S 2$.

If $G=\mathscr{D} \cap G_{0}$ is furnished with the topology and uniform structure induced by the field topology and field uniformity of $\mathscr{D}$ and if $p$ is the restriction of $\rho: \mathscr{D} \rightarrow T$ to $G$, then $(G, p, T)$ is also 
a uniform field.

3.9. Suppose that $\Sigma$ is the algebra $\Gamma^{b}(\pi)$ of all bounded global sections of $(E, \pi, T)$. If $\lambda$ and $\mu$ are global sections in $(G, p, T)$, then $u \mapsto(\lambda \mu)(t)=\lambda(t) \mu(t)$ is also a global section in $(G, p, T)$.

Proof. For every $\sigma \in \Sigma, t \mapsto \mu(t) \sigma(t)$ belongs to $\Sigma$ by 2.2 and then $t \mapsto \lambda(t)(\mu(t) \sigma(t))$ is also in $\Sigma$, then invoking 2.2 again, $\lambda \mu$ is a global section in $(G, p, T)$.

3.10. The function $u \mapsto u^{-1}: G \mapsto G$ is continuous when $G$ is furnished with its field topology.

Proof. Let $u$ be an element of $G$ and set $t=p(u)$. Given a neighborhood of $u^{-1}$ in $G$ of the form $M\left(u^{-1}, \sigma, \tau, Q, \varepsilon\right)$ where $\sigma, \tau \in \Sigma$ and $u^{-1} \sigma(t)=\tau(t)$, then $u \tau(t)=\sigma(t)$ and so $M(u, \tau, \sigma, Q, \varepsilon)$ is a neighborhood of $u$ in $G$. If $v \in M(u, \tau, \sigma, Q, \varepsilon)$, then $p\left(v^{-1}\right)=p(v)$ belongs to $Q$ and $\|v \tau(p(v))-\sigma(p(v))\|=\left\|v^{-1} \sigma(p(v))-\tau(p(v))\right\|<\varepsilon$, hence $v^{-1} \in M\left(u^{-1}, \sigma, \tau, Q, \varepsilon\right)$, therefore $u \mapsto u^{-1}: G \rightarrow G$ is continuous.

3.11. The function $(u, v) \mapsto u v: G \vee G \rightarrow G$ is continuous if $\Sigma=\Gamma^{b}(\pi)$.

Proof. Given an element $\left(u_{0}, v_{0}\right)$ of $G \vee G$, choose any neighborhood $U(\sigma, \varepsilon)(\gamma)$ and set $t_{0}=p\left(u_{0} v_{0}\right)$. Without loss of generality $\gamma$ can be taken of the form $\gamma=\xi \eta$ where $\xi$ and $\eta$ are local sections in $(G, p, T)$ such that $\xi\left(p\left(u_{0}\right)\right)=u_{0}$ and $\eta\left(p\left(v_{0}\right)\right)=v_{0}$. We can consider $\eta$ as the restriction of a global section $\bar{\eta}$ to an open subset of $T$. Since $\sigma$ belongs to $\Sigma$, then $\bar{\eta} \sigma$ belongs to $\Sigma$ too, and if $u$ is in $U(\bar{\eta} \sigma, \varepsilon / 2)(\xi)$ and $v$ is in $U(\sigma, \varepsilon / 2)(\eta)$, then $u v$ is in $U(\sigma, \varepsilon)(\gamma)$.

3.12. Condition $\left(\mathrm{b}^{\prime}\right)$ of 3.3 is satisfied by $(G, p, T)$, that is, a fundamental system of entourages for the field uniformity $\mathscr{Q}$ of $(G, p, T)$ is given by the sets of the form $\left\{(u, v) \in G \vee G \mid u^{-1} v \in W(\zeta)\right\}$ where $\zeta$ is the unit element of $\Gamma(p)$ and $W$ range through $\mathscr{U}$.

Proof. For a fundamental entourage of $\mathscr{C}$ one has

$$
\begin{aligned}
U(\sigma, \varepsilon) & =\{(u, v) \in G \vee G \mid\|u \sigma(p(u))-v \sigma(p(v))\|<\varepsilon\} \\
& =\left\{(u, v) \in G \vee G \mid\left\|\sigma(p(u))-u^{-1} v \sigma(p(v))\right\|<\varepsilon\right\} \\
& =\left\{(u, v) \in G \vee G \mid u^{-1} v \in U(\sigma, \varepsilon)(\zeta)\right\} .
\end{aligned}
$$

Proposition 3.13. If $\Sigma=\Gamma^{b}(\pi)$, then $(G, p, T)$ is a left uniform 
field of topological groups and hence $\Gamma(p)$ is a topological group equipped with the standard topology derived from $(G, p, T)$.

Proof. Condition (a) of Definition 3.1 was proved in 3.10 and 3.11 , condition $\left(b^{\prime}\right)$ of Remark 3.3 was proved in 3.12 ; we are going to verify condition (d) of Definition 3.1.

Given an entourage of the form $U(\sigma, \varepsilon)$ and a global section $\lambda$ of $(G, p, T)$, we take $V=U\left(\lambda^{-1} \sigma, \varepsilon\right)$, then $(u, v) \in V$ implies $\left(\lambda(t) u \lambda(t)^{-1}\right.$, $\left.\lambda(t) v \lambda(t)^{-1}\right) \in U(\sigma, \varepsilon)$, with $t=p(u)=p(v)$. Hence $(G, p, T)$ is a left uniform field of topological groups.

\section{Automorphisms leaving center fixed.}

4.1. Let $(E, \pi, T)$ be a field of involutive normed algebras and $\Sigma=\Gamma^{b}(\pi)$, then an isometric star automorphism $\lambda$ of $\Sigma$ is $C^{b}(T)$ linear if and only if it leaves setwise invariant the ideals of the form $I_{t}=\{\sigma \in \Sigma \mid \sigma(t)=0\}$, i.e. $\lambda I_{t} \subset I_{t}$ for each $t \in T$. In particular, $\lambda I_{t} \subset I_{t}$ implies $\lambda^{-1} I_{t} \subset I_{t}$ and hence $\lambda I_{t}=I_{t}$.

\section{Proof. This is a direct consequence of [8] 2.3.}

THEOREM 4.2. Let $(E, \pi, T)$ be a field of involutive normed algebras and $\Sigma=\Gamma^{b}(\pi)$. The group $N$ of isometric star automorphisms of $\Sigma$ leaving setwise invariant the ideals of the form $I_{t}=$ $\{\sigma \in \Sigma \mid \sigma(t)=0\}$ is isomorphic to the group $\Gamma(p)$ of all global sections of the field of isometries $(G, p, T)$ associated with $(E, \pi, T)$.

Proof. Let $\lambda \in N$ and $t \in T$. Define $\lambda_{t}$ as follows $x \mapsto \lambda_{t}(x)=$ $\left(\lambda \sigma_{x}\right)(t): E_{t} \rightarrow E_{t}$ where $\sigma_{x} \in \Sigma$ and $\sigma_{x}(t)=x$. The definition of $\lambda_{t}(x)$ is clearly independent of the particular $\sigma_{x}$ taken through $x$, and $\lambda_{t}$ is a star automorphism of $E_{t}$ whose inverse is $\left(\lambda^{-1}\right)_{t}$. Furthermore, by [8] 2.3, $\left\|\lambda_{t}(x)\right\|=\left\|\left(\lambda \sigma_{x}\right)(t)\right\| \leqq\|\lambda\|\left\|\sigma_{x}(t)\right\|=\left\|\sigma_{x}(t)\right\|=\|x\|$ and $\left\|\lambda_{t}^{-1}(x)\right\|=\left\|\left(\lambda^{-1} \sigma_{x}\right)(t)\right\| \leqq\left\|\lambda^{-1}\right\|\left\|\sigma_{x}(t)\right\|=\left\|\sigma_{x}(t)\right\|$, if $x=\sigma(t)$ for each $x \in E_{t}=\pi^{-1}(t)$, then $\|x\|=\left\|\lambda_{t}^{-1}\left(\lambda_{t}(x)\right)\right\| \leqq\left\|\lambda_{t}(x)\right\|$, hence $\lambda_{t}$ is an isometry of $E_{t}$.

Consider the selection $\varphi(\lambda)$ of $(G, p, T)$ given by $t \mapsto \lambda_{t}: T \rightarrow G$. For every $\sigma \in \Sigma$ the selection $t \mapsto \lambda_{t}(\sigma(t)): T \rightarrow E$ is continuous, hence by $2.4 \varphi(\lambda)$ is a global section. Thus $\varphi$ is a group homomorphism from $N$ into $\Gamma(p)$. On the other hand, given a global section $\eta$ in the field of automorphisms $(G, p, T)$ and a section $\sigma \in \Sigma$, the map $t \mapsto \eta(t) \sigma(t): T \rightarrow E$ is a section $\eta \sigma \in \Sigma$, and since $\|(\eta \sigma)(t)\|=\|\sigma(t)\|$ for every $t \in T$, then $\alpha(\eta): \sigma \mapsto \eta \sigma$ is an isometric star automorphism of $\Sigma$ leaving setwise invariant the ideals $\left\{I_{t} \mid t \in T\right\}$ and $\alpha$ is a group 
homomorphism whose inverse is $\varphi$, indeed, for every $\lambda \in N, \sigma \in \Sigma$ and $t \in T, \quad(\alpha(\varphi(\lambda)) \sigma)(t)=\varphi(\lambda)(t) \sigma(t)=(\lambda \sigma)(t)$, then $\alpha(\varphi(\lambda))=\lambda$, that is, $\alpha \circ \varphi=\mathrm{id}_{N}$. On the other hand, for every $\eta \in \Gamma(p)$, every $t \in T$ and every $x \in E_{t}$, if $\sigma \in \Sigma$ and $\sigma(t)=x$, then $\varphi(\alpha(\eta))(t) x=\varphi(\alpha(\eta))(t) \sigma(t)=$ $(\alpha(\eta) \sigma)(t)=\eta(t) \sigma(t)=\eta(t) x$, then $\alpha(\varphi(\eta))(t)=\eta(t)$, therefore $\varphi(\alpha(\eta))=\eta$, that is, $\varphi \circ \alpha=\mathrm{id}_{\Gamma(p)}$.

REMARK 4.3. Consider the group homomorphism $\varepsilon_{t}: \eta \mapsto \eta(t)$ : $\Gamma(p) \rightarrow$ Aut $\pi^{-1}(t)$, then $\operatorname{Im} \varepsilon_{t}=p^{-1}(t)$ because $G$ was defined as the subset of $G_{0}$ consisting of the points $u$ such that there is a global section $\eta: T \rightarrow G_{0}$ with $\eta(p(u))=u$. But

$$
\begin{aligned}
\operatorname{ker} \varepsilon_{t} & =\{\eta \in \Gamma(p) \mid(\forall \sigma \in \Sigma)(\eta(t) \sigma(t)=\sigma(t))\} \\
& =\left\{\eta \in \Gamma(p) \mid(\forall \sigma \in \Sigma)\left(\alpha(\eta) \sigma-\sigma \in I_{t}\right)\right\},
\end{aligned}
$$

then $p^{-1}(t)$ is isomorphic to the quotient proup $N / R_{t}$ where $R_{t}=$ $\left\{\lambda \in N \mid(\forall \sigma \in \Sigma)\left(\lambda \sigma-\sigma \in I_{t}\right)\right\}$.

4.4. Let $A$ be a $C^{*}$-algebra with identity and $B$ a sub- $C^{*}$ algebra of $A$ contained in its center and containing the identity, then the group $N$ of star automorphisms of $A$ leaving setwise invariant the closed two sided ideals generated by a maximal ideal of $B$ is equal to the group of star automorphisms of $A$ leaving $B$ elementwise fixed.

\section{Proof. This follows from 4.1 and [8], Theorem 3.5.}

Definition 4.5. Let $(E, \pi, T)$ be a field of involutive normed algebras, an automorphism $(\ell, \Lambda)$ of $(E, \pi, T)([8], 4.3)$ is called isometric star automorphism if $\ell$ is the identity map of $T$ and $\Lambda: E \rightarrow E$ is a homeomorphism such that $\pi \circ \Lambda=\pi$ and $x \mapsto \Lambda x: E_{t} \rightarrow E_{t}$ is an isometric star automorphism.

4.6. Let $(E, \pi, T)$ be a field of involutive normed algebras and $\Sigma=\Gamma^{b}(\pi)$, then the group $N$ of isometric star automorphisms of $\Sigma$ leaving setwise invariant the ideals of the form $I_{t}=\{\sigma \in \Sigma \mid \sigma(t)=0\}$ is anti-isomorphic to the group $\operatorname{Isom}(E, \pi, T)$ of isometric star automorphisms of the field $(E, \pi, T)$.

Proof. Let $\xi$ be an element of $N$. Define $\theta(\xi)$ by $x \mapsto \theta(\xi) x=$ $(\xi \sigma)(\pi(x)): E \rightarrow E$ with $\sigma \in \Sigma$ such that $\sigma(\pi(x))=x$. By [8] 2.3, $\|\theta(\xi) x\|=\|(\xi \sigma)(\pi(x))\| \leqq\|\xi\|\|\sigma(\pi(x))\|=\|x\|$.

Similarly $\left\|\theta\left(\xi^{-1}\right) x\right\| \leqq\|x\|$ for every $x \in E$ and since $\theta\left(\xi^{-1}\right)=$ $\theta(\xi)^{-1}$, then $\|x\|=\left\|\theta\left(\xi^{-1}\right) \theta(\xi) x\right\| \leqq\|\theta(\xi) x\|$, that is $\theta(\xi)$ is an isometric 
star automorphism in each stalk for every $\xi \in N$.

Thus $\left(1_{T}, \theta(\xi)\right)$ belongs to Isom $(E, \pi, T)$.

Conversely, if $\left(1_{T}, \Lambda\right)$ is an isometric star automorphism of $(E, \pi, T)$ and $\sigma$ is an element of $\Sigma$, then $\Lambda \circ \sigma$ is also an element of $\Sigma$. Define $\gamma(\Lambda)$ as follows $\sigma \mapsto \gamma(\Lambda) \sigma=\Lambda \circ \sigma: \Sigma \rightarrow \Sigma$, this is an isometric star automorphism of $\Sigma$ and leaves the ideals $\left\{I_{t} \mid t \in T\right\}$ invariant. Thus $\gamma:\left(1_{T}, \Lambda\right) \mapsto \gamma(\Lambda)$ is a group homomorphism from Isom $(E, \pi, T)$ into the opposite group of $N$. Furthermore, for every $x \in E$, if $\sigma \in \Sigma$ and $\sigma(\pi(x))=x$, then $\theta(\gamma(\Lambda)) x=(\gamma(\Lambda) \sigma)(\pi(x))=$ $(\Lambda \circ \sigma)(\pi(x))=\Lambda(\sigma(\pi(x)))=\Lambda x$, hence $\theta(\gamma(\Lambda))=\Lambda$ for every $\left(1_{T}, \Lambda\right) \in$ $\operatorname{Isom}(E, \pi, T)$.

Similarly for every $t \in T, \quad \sigma \in \Sigma$ and $\xi \in N, \quad \gamma(\theta(\xi) \sigma)(t)=$ $(\theta(\xi) \circ \sigma)(t)=\theta(\xi)(\sigma(t))=(\xi \sigma)(t)$, then $\gamma(\theta(\xi))=\xi$ for every $\xi \in N$. Thus $\gamma$ is an anti-isomorphism between $\operatorname{Isom}(E, \pi, T)$ and $N$ whose inverse is $\theta$.

4.7. For a $C^{*}$-algebra with identity, we collect the preceding results in the following

Theorem. Let $A$ be a $C^{*}$-algebra with identity and Aut $A$ its group of star automorphisms. There is an exact sequence

$$
1 \longrightarrow N \stackrel{j}{\longrightarrow} \text { Aut } A \stackrel{k}{\longrightarrow} H
$$

where $H$ is the group of homeomorphisms of the center $Z$ of $A$, and $k$ assigns to a star automorphism of $A$ the homeomorphism of $T$ associated with the inverse of its restriction to $Z$.

For $N$ one can take of the follouing groups:

The group $N_{1}$ of star automorphisms of $A$ leaving the center elementwise fixed.

The group $\mathrm{N}_{2}$ of star automorphisms of $A$ leaving setwise invariant the closed two sided ideals generated by a maximal ideal of the center $Z$ of $A$.

The groups $N_{3}$ of all global sections of the field $(G, p, T)$ obtained from the canonical field $(E, \pi, T)$, over the spectrum $T$ of $Z$, associated $A$.

The group $N_{4}$ opposite to the group of star automorphisms of the canonical field $(E, \pi, T)$ of the form $\left(1_{T}, \Lambda\right)$ where $1_{T}$ is the identity map of the spectrum $T$ of the center of $A$.

Proof. This follows from 4.2, 4.4, 4.6 and [8] Theorem 3.5.

4.8. Let $(E, \pi, T)$ be a field of $C^{*}$-algebras with identity, Aut $(E, \pi, T)$ the group of star automormorphisms of $(E, \pi, T)$ and 
$\operatorname{Aut}_{T}(E, \pi, T)$ its group of star automorphisms of the form $\left(1_{T}, \Lambda\right)$, then we have the exact sequence

$$
\operatorname{Aut}_{T}(E, \pi, T) \stackrel{\chi}{\longrightarrow} \operatorname{Aut}(E, \pi, T) \stackrel{\kappa}{\longrightarrow} H
$$

where $\chi$ is the inclusion map, $H$ is the group of homeomorphisms of $T$ and $\kappa(\ell, \Lambda)=\ell$.

Furthermore, if $(E, \pi, T)$ is a central field, that is, if the center of $\Gamma(\pi)$ is $C(T)$, then $\operatorname{Aut}(E, \pi, T)$ is anti-isomorphic to Aut $\Gamma(\pi)$.

Proof. Every star automorphism of $\Gamma(\pi)$ leaves its center invariant, then if $(E, \pi, T)$ is central it determines a star automorphism of the pair $(C(T), \Gamma(\pi))$ and hence a star automorphism of $(E, \pi, T)$, conversely every star automorphism of $(E, \pi, T)$ determines a star automorphism of $(C(T), \Gamma(\pi))$ and hence a star automorphism of $\Gamma(\pi)([8], 4.6)$.

\section{Fields of derivations.}

Definition 5.1. Let $A$ be a complex algebra and $\delta: A \rightarrow A$ be a linear map, $\delta$ is a derivation of $A$ provided $\delta(x y)=\delta(x) y+x \delta(y)$ for every $x, y \in A$, Given $a \in A$, the map $x \mapsto a x-x a$ is an inner derivation. If $\delta$ and $\rho$ are derivations of $A$ and $z$ is a complex number, then $x \mapsto \delta(x)+\rho(x), x \mapsto z \delta(x)$ and $x \mapsto \delta(\rho(x))-\rho(\delta(x))$ are derivations of $A$ denoted by $\delta+\rho, z \delta$ and $[\delta, \rho]$ respectively. The set $J$ of all derivations of $A$ is a Lie Algebra with respect to these operations. In particular the bracket product is a bilinear map such that $[\lambda, \mu]=-[\mu, \lambda]$ and $[[\lambda, \mu], \nu]+[[\mu, \nu], \lambda]+[[\nu, \lambda], \mu]=0$. If $\delta$ is a derivation of a $C^{*}$-algebra, then $\delta$ is norm continuous and its restriction to the center of $A$ is identically zero.

5.2. If $A$ is a $C^{*}$-algebra and $I$ is a closed two sided ideal of $A$, then $\delta(I) \subset I$ for every derivation $\delta$ of $A$ ([5], 2.2).

Proof. $I^{2}=I$, where $I^{2}$ denotes the linear subspace generated by the set of all products $x y$, with $x \in I$ and $y \in I$. Since $\delta(x y)=$ $x \delta(y)+\delta(x) y$ belongs to $I$, for every $x, y \in I$, then $\delta(I) \subset I$.

5.3. Let $(E, \pi, T)$ be a field of normed algebras. Denote by Der $E_{t}$ the Lie algebra of all derivations of $E_{t}=\pi^{-1}(t)$ and let $L_{0}$ be the disjoint union of the family $\left\{\right.$ Der $\left.E_{t} \mid t \in T\right\}$. Let $\Sigma$ be a normed algebra consisting of a full set of bounded global sections of $(E, \pi, T)$ and consider again the uniform field $(\mathscr{D}, \rho, T)$ defined in $\S 2$, associated with $\Sigma$. 
If $L=\mathscr{D} \cap L_{0}$ is equipped with the topology and uniform structure induced by the field topology and field uniformity of $\mathscr{D}$ and if $q$ is the restriction of $\rho: \mathscr{D} \rightarrow T$ to $L$, then $(L, q, T)$ is also a uniform field.

5.4. Suppose that $\Sigma$ consists of all global sections with quasicompact support in $(E, \pi, T)$ and that $\lambda$ and $\mu$ are global sections in $(L, q, T)$, then $t \mapsto(z \lambda)(t)=z \lambda(t)$ with $z$ a complex number, $t \mapsto(\lambda+\mu)(t)=\lambda(t)+\mu(t)$ and $t \mapsto[\lambda, \mu](t)=[\lambda(t), \mu(t)]$ are also global sections of $(L, q, T)$.

Proof. If $\sigma \in \Sigma$, then by $2.4 t \mapsto \lambda(t) \sigma(t)$ and $t \mapsto \mu(t) \sigma(t)$ are global sections of $(E, \pi, T)$ and their supports are still quasicompact.

The maps $t \mapsto(z \lambda(t))(\sigma(t)), t \mapsto(\lambda(t)+\mu(t))(\sigma(t))$ and

$$
t \longmapsto \lambda(t)(\mu(t) \sigma(t))-\mu(t)(\lambda(t) \sigma(t))
$$

also belong to $\Sigma$, thus by $2.4, z \lambda, \lambda+\mu$ and $[\lambda, \mu]$ are global sections in the field $(L, q, T)$.

REMARK. Recall that if $\sigma$ is a global section with quasicompact support the upper semicontinuity of the function $t \mapsto\|\sigma(t)\|: t \rightarrow \boldsymbol{R}_{+}$ implies that $\sigma$ is bounded (1.9).

5.5. In the field of derivations $(L, q, T)$ the $\operatorname{sum}(u, v) \mapsto$ $u+v: L \vee L \rightarrow L$ is continuous.

Proof. Let $\left(u_{0}, v_{0}\right) \in L \vee L, t=q\left(u_{0}\right)=q\left(v_{0}\right)$ and $M\left(u_{0}+v_{0}, \sigma, \tau\right.$, $Q, \varepsilon)$ a fundamental neighborhood of $u_{0}+v_{0} \quad$ Without loss of generality $\tau$ can be taken of the form $\tau=\tau_{1}+\tau_{2}$ where $\tau_{1}$ and $\tau_{2}$ are global sections such that $\tau_{1}(t)=u_{0} \sigma(t)$ and $\tau_{2}(t)=v_{0} \sigma(t)$. If $(u, v) \in L \vee L$, $u \in M\left(u_{0}, \sigma, \tau_{1}, Q, \varepsilon / 2\right)$ and $v \in M\left(v_{0}, \sigma, \tau_{2}, Q, \varepsilon / 2\right)$, the $u+v \in M\left(u_{0}+v_{0}\right.$, $\sigma, \tau, Q, \varepsilon)$. Hence the sum is continuous.

5.6. Let $(E, \pi, T)$ be a field of normed algebras, $\Gamma_{c}(\pi)$ the algebra of all global sections with quasicompact support of $(E, \pi, T)$ and $J$ the Lie algebra of derivations of $\Gamma_{\mathrm{c}}(\pi)$ leaving setwise invariant the ideals of the form $I_{t}=\left\{\sigma \in \Gamma_{c}(\pi) \mid \sigma(t)=0\right\}$, then $J$ is isomorphic to the algebra $\Gamma(q)$ of all global sections of the field $(L, q, T)$.

Proof. Let $\lambda \in J$ and $t \in T$. Define $\lambda_{t}$ as follows $\lambda_{t}: x \mapsto$ $\left(\lambda \sigma_{x}\right)(t): E_{t} \rightarrow E_{t}$ where $\sigma_{x} \in \Gamma_{c}(\pi)$ and $\sigma_{x}(t)=x$. This function is well defined, indeed, if $\sigma, \tau \in \Gamma_{c}(\pi)$ and $\sigma(t)=\tau(t)=x$, then $\lambda(\sigma-\tau) \in I_{t}$ 
and $(\lambda \sigma)(t)=(\lambda \tau)(t)$. Let $\psi(\lambda): t \mapsto \lambda_{t}: T \rightarrow L$, if $\sigma \in \Gamma_{c}(\pi)$, then $t \mapsto$ $\lambda_{t}(\sigma(t)): T \rightarrow E$ is a section, hence by $2.4 \psi(\lambda)$ is an isomorphism from the Lie algebra $J$ into the Lie algebra of all global sections of $(L, q, T)$.

On the other hand, given a global section $\xi$ in the field $(L, q, T)$ and a global section $\sigma \in \Gamma_{c}(\pi), \beta(\xi) \sigma: t \mapsto \xi(t) \sigma(t): T \rightarrow E$ belongs to $\Gamma_{\mathrm{o}}(\pi)$. Thus $\beta: \xi \mapsto \beta(\xi): \Gamma(q) \rightarrow J$ is an isomorphism of Lie algebras whose inverse is $\psi$.

5.7. With the same hypothesis and notation of 5.6, let $\varepsilon_{t}$ be the Lie algebra homomorphism $\varepsilon_{t}: \xi \mapsto \xi(t): \Gamma(q) \rightarrow$ Der $E_{t}$, then $\operatorname{Im} \varepsilon_{t}=q^{-1}(t)$ because $L$ was defined as the subset of $L_{0}$ consisting of the points $u$ such that there is a global section $\xi: T \rightarrow L_{0}$ with $\xi(q(u))=u$. But

$$
\begin{aligned}
\operatorname{ker} \varepsilon_{t} & =\left\{\xi \in \Gamma(q) \mid\left(\forall \sigma \in \Gamma_{c}(\pi)\right)(\xi(t) \sigma(t)=0)\right\} \\
& =\left\{\xi \in \Gamma(q) \mid\left(\forall \sigma \in \Gamma_{c}(\pi)\right)\left(\beta(\xi) \sigma \in I_{t}\right)\right\},
\end{aligned}
$$

then the fiber $L_{t}=q^{-1}(t)$ is isomorphic to the quotient Lie algebra $J / K_{t}$ where $K_{t}=\left\{\lambda \in J \mid\left(\forall \sigma \in \Gamma_{c}(\pi)\right)\left(\lambda \sigma \in I_{t}\right)\right\}$.

DEFINITION 5.8. Let $(E, \pi, T)$ be a field of normed algebras. $A$ continuous map $\Delta: E \rightarrow E$ is called a derivation of $(E, \pi, T)$ if $\pi \circ \Delta=\pi$ and for every $t \in T$, the map $x \mapsto \Delta x: E_{t} \rightarrow E_{t}$ is a derivation of $E_{t}=\pi^{-1}(t)$.

5.9. Let $(E, \pi, T)$ be a field of normed algebras and $\Gamma_{0}(\pi)$ the algebra of all global sections with quasicompact support, then the Lie algebra $J$ of all continuous derivations of $\Gamma_{c}(\pi)$ leaving setwise invariant the ideals $I_{t}=\left\{\sigma \in \Gamma_{c}(\pi) \mid \sigma(t)=0\right\}$ for every $t \in T$ is isomorphic to the Lie algebra $\operatorname{Der}(E, \pi, T)$ of derivations of the field $(E, \pi, T)$.

Proof. Let $\rho$ be an element of $J$. Define $F(\rho)$ as follows $x \mapsto F(\rho) x=(\rho \sigma)(\pi(x)): E \rightarrow E$, where $\sigma$ is an element of $\Gamma_{c}(\pi)$ such that $\sigma(\pi(x))=x$. The function $F(\rho)$ is well defined because if $\tau(\pi(x))=x$ and $t=\pi(x)$, then $\sigma-\tau \in I_{t}$ and hence $(\rho \sigma)(\pi(x))=$ $(\rho \tau)(\pi(x))$.

To prove that $F(\rho)$ is continuous, say at $x$, let $\mathscr{T}(\tau, \varepsilon) \cap \pi^{-1}(Q)$ be a fundamental neighborhood of $F(\rho) x$, without loss of generality one can take $\tau$ of the form $\tau=\rho \sigma$ where $\sigma$ is an element of $\Gamma_{c}(\pi)$ such that $\sigma(\pi(x))=x$.

Take $W=\mathscr{T}\left(\sigma, \varepsilon(\varepsilon+\|\rho\|)^{-1}\right) \cap \pi^{-1}(Q)$ as neighborhood of $x$. By ([8], 2.3), for every $y \in W$, 


$$
\|F(\rho) y-\tau(\pi(y))\| \leqq\|\rho\|\|y-\sigma(\pi(y))\|<\varepsilon,
$$

then $F(\rho) y \in \mathscr{T}(\tau, \varepsilon) \cap \pi^{-1}(Q)$ and $F(\rho)$ is continuous at $x$, thus $F(\rho)$ belongs to $\operatorname{Der}(E, \pi, T)$ and $F$ is a Lie algebra homomorphism. Conversely, if $\Delta$ is a derivation of $(E, \pi, T)$ and $\sigma \in \Gamma_{c}(\pi)$, then $\Delta \circ \sigma$ is also a global section with quasicompact support in the field $(E, \pi, T)$.

Define $G(\Delta)$ by $\sigma \mapsto G(\Delta) \sigma=\Delta \circ \sigma: \Gamma_{c}(\pi) \rightarrow \Gamma_{c}(\pi)$, then $G(\Delta)$ is a derivation of $\Gamma_{c}(\pi)$ leaving invariant the ideals of the form $I_{t}$. Furthermore $G(\Delta)$ is a continuous function, indeed, let $\sigma \in \Gamma_{c}(\pi), T_{o}$ the support of $\sigma$ and $\varepsilon>0$. Since for every $t \in T, \Delta$ is continuous at $\sigma(t)$, there is a neighborhood $Q_{t}$ of $t$ and $\delta_{t}>0$ such that $x \in$ $\pi^{-1}\left(Q_{t}\right) \cap \mathscr{T}\left(\sigma, \delta_{t}\right)$ implies $\Delta x \in \mathscr{T}(\Delta \sigma, \varepsilon)$. Without loss of generality $Q_{t}$ can be supposed to contain the complement of $T_{o}$. Since $T_{\sigma}$ is quasicompact, there exist a finite number of $Q_{t}$ 's covering $T$. If $\delta$ is taken to be the minimum of the corresponding $\delta$ 's, then $\triangle \mathscr{T}(\sigma, \delta) \subset$ $\mathscr{T}(\Delta \sigma, \varepsilon)$. It follows that $G(\Delta)$ is continuous and hence it belongs to $J$.

Therefore $G$ is an isomorphism between $\operatorname{Der}(E, \pi, T)$ and $J$ whose inverse is $F$.

\subsection{Summing up, we have the following}

Theorem. If $A$ is $C^{*}$-algebra with identity and $B$ is a sub-C*algebra contained in the center of $A$ and containing its identity, then the following Lie algebras are isomorphic.

(i) The Lie algebra of derivations of $A$.

(ii) The Lie algebra of derivations of the canonical field $(E, \pi, T)$ associated with the pair $(B, A)$.

(iii) The Lie algebra of global sections of the field of derivations $(L, q, T)$ obtained from $(E, \pi, T)$.

Proof. This follows from 5.2, 5.6 and 5.9.

\section{The exponential function.}

6.1. Let $A$ be a $C^{*}$-algebra with identity. We identify $A$ with the algebra $\Gamma(\pi)$ of all global sections of the canonical field $(E, \pi, T)$ associated with $A$ over the spectrum $\mathrm{T}$ of the center of $A$. As usual $I_{t}$ denotes the ideal $I_{t}=\{\sigma \in \Gamma(\pi) \mid \sigma(t)=0\}$. It was observed in [8] 3.1, that $I_{t}$ is the closed two sided ideal generated by $t \in T$.

Let $J$ be the Lie algebra of derivations of $A$ and $N$ the group of automorphisms of $A$ leaving the center elementwise fixed. The following diagram is commutative 


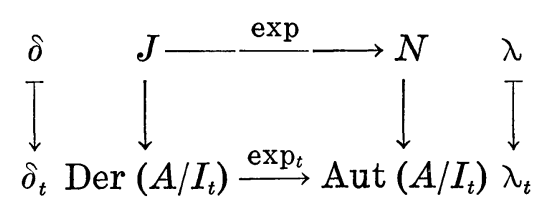

where $\delta_{t}(x)=(\delta \sigma)(t), \lambda_{t}(x)=(\lambda \sigma)(t)$ for every $x \in A / I_{t}$ with $\sigma \in \Gamma(\pi)$ such that $\sigma(t)=x,(\exp \delta)(a)=a+\delta(a)+\delta^{2}(a) / 2 !+\cdots$, for every $\delta \in J$ with $a \in A$, and

$$
\left(\exp _{t} u\right)\left(a+\mathrm{I}_{t}\right)=\left(a+I_{t}\right)+u\left(a+I_{t}\right)+u^{2}\left(a+I_{t}\right) / 2 !+\cdots,
$$

for every $u \in \operatorname{Der}\left(A / I_{t}\right)$ and every $a \in A$.

Proof. $\delta_{t}$ and $\lambda_{t}$ are well defined because the ideal $I_{t}$ is left fixed by $\delta \in J$ and $\lambda \in N$.

Let $\delta \in J$, then $\left(\exp \delta_{t}\right)(x)=x+\delta_{t}(x)+\delta_{t}^{2}(x) / 2 !+\cdots=\sigma(t)+$ $(\delta \sigma)(t)+\left(\delta^{2} \sigma\right)(t) / 2 !+\cdots=((\exp \delta) \sigma)(t)=(\exp \delta)_{t}(x)$, where $x \in A / I_{t}$ and $\sigma(t)=x$. Thus the diagram is commutative.

6.2. Now we can define an "exponential function" from the field $(L, q, T)$ of derivations of $A=\Gamma(\pi)$ into the field $(G, p, T)$ of automorphisms of $A$.

$$
E X P: u \longmapsto(\exp )_{t}(u): L \longrightarrow G \text { with } t=q(u) .
$$

Clearly $p(E X P u)=q(u)$ for every $u \in L$, and using the notation of 5.6 from $E X P: L \rightarrow G$ we get back exp: $J \rightarrow N$ as follows: Consider $\sigma \mapsto(\operatorname{EXP}(\psi(\delta)(\cdot)))(\sigma(\cdot))$, with $\delta \in J$ and $\sigma \in \Gamma(\pi)$, then

$$
(E X P(\psi(\delta)(t)))(\sigma(t))=\left(\exp _{t} \delta_{t}\right)(\sigma(t))=(\exp \delta)_{t}(\sigma(t))=((\exp \delta) \sigma)(t)
$$

for every $t \in T$. Thus $\operatorname{EXP}(\psi(\delta)(\cdot))(\sigma(\cdot))=(\exp \delta) \sigma$.

6.3. EXP: $L \rightarrow G$ is continuous on bounded sets when $L$ and $G$ are endowed with their field topologies.

Proof. Let $L_{M}=\{w \in L\|\| w \| \leqq M\}$ where $\|w\|$ is the operator norm of $w$. Let $u \in L_{M}, t=q(u), \varepsilon>0$ and $\delta \in J$ such that $\delta_{t}=u$. Since $\operatorname{EXP} u=\exp _{t} u=\exp _{t} \delta_{t}=(\exp \delta)_{t}$, then a fundamental neighborhood of $E X P u$ in $G$ is of the form

$$
\begin{array}{r}
\left\{v \in G \mid s=p(v) \in Q \& \inf _{x \in I_{s}}\|\lambda a-(\exp \delta) a+x\|<\varepsilon\right. \\
\text { for } \left.\lambda \in N \text { with } \lambda_{s}=v\right\}
\end{array}
$$

where $Q$ is an open neighborhood of $t=p(E X P u)$ and $a \in A$. Let $k$ be a positive integer such that $2 M\|a\| \sum_{n=k}^{\infty} M^{n} / n !<\varepsilon / 2$ and consider the following neifhborhood of $u$ in $L_{M}$. 


$$
\begin{array}{r}
V(u)=\left\{w \in L_{M} \mid s=q(w) \& \inf _{x \in I_{s}}\left\|(\xi-\delta) \delta^{j} a+x\right\|<\varepsilon / 2 \exp (M+1)\right. \\
\text { for } \left.j=1, \cdots k \& \xi \in J \text { with } \xi_{s}=w\right\}
\end{array}
$$

Denote by $\|\cdot\|_{s}$ the quotient norm in $A / I_{s}$, then for every $w=\xi_{s} \in V(u)$ we have

$$
\begin{aligned}
& \left\|(\exp \xi) a-(\exp \delta) a+I_{s}\right\|_{s} \\
\leqq & \sum_{n=1}^{\infty} 1 / n !\left\|\left(\xi^{n}-\delta^{n}\right) a+I_{s}\right\|_{s} \\
= & \sum_{n=1}^{\infty} 1 / n !\left\|\sum_{i=1}^{n} \xi^{i-1}(\xi-\delta) \delta^{n-i} a+I_{s}\right\|_{s} \\
\leqq & \sum_{n=1}^{k}(M+1)^{n-1} /(n-1) ! \sup _{1 \leq i \leq n}\left\|(\xi-\delta) \delta^{n-i} a+I_{s}\right\|_{s} \\
& +\sum_{n=k+1}^{\infty} n M^{n-1} / n !\left\|\xi_{s}-\delta_{s}\right\|\left\|a+I_{s}\right\|_{s} \\
\leqq & \varepsilon / 2+2 M\|a\| \sum_{n=k}^{\infty} M^{n} / n ! \leqq \varepsilon .
\end{aligned}
$$

Thus $E X P: L \rightarrow G$ is continuous on bounded sets.

7. One example. Let $M_{2}(C)$ be the algebra of all $2 \times 2$ complex matrices and let $A$ be the $C^{*}$-algebra consisting of all sequences $X=\left(X_{n}\right)_{n}$ of elements of $M_{2}(C)$ converging to a diagonal matrix

$$
\left(\begin{array}{cc}
\alpha(X) & 0 \\
0 & \beta(X)
\end{array}\right)
$$

The center $Z$ of $A$ is $C^{*}$-isomorphic to the algebra $c$ of all convergent sequences of complex numbers. For every positive integer $k \geqq 1$ (resp. for $k=0$ ) the ideal $c_{k}$ of all convergent complex sequences whose $k$ th term is zero (resp. whose limit is zero) is a maximal ideal of $\mathfrak{c}$, and these are all its maximal ideals.

The closed two sided ideal generated by $c_{k}$ with $k \geqq 1$ (resp. by $\mathfrak{c}_{0}$ ) is the ideal of all $X$ such that $X_{k}=0$ (resp. $\lim X_{k}=0$ ). By the (noncommutative) Gelfand-Naimark representation theorem ([2], Chapter III, 8.14; [8], 3.5), $A$ is isometrically star isomorphic to the algebra of all global sections of a field of $C^{*}$-algebra $(E, \pi, T)$ whose base space $T=Z_{+} \cup\{\omega\}$ is the one point compactification of the discrete space $\boldsymbol{Z}_{+}$of position integers, and whose stalks are $\{t\} \times M_{2}(C)$ for $t \neq \omega$ and $\{t\} \times C^{2}$ for $t=\omega$. Every star automorphism of $M_{2}(C)$ is inner given by a unitary matrix, and $C^{2}$ has only two automorphisms, the identity and $\left(z_{1}, z_{2}\right) \mapsto\left(z_{2}, z_{1}\right): C^{2} \mapsto C^{2}$. The group $N$ of star automorphisms of $A$ leaving the center elementwise fixed is then isomorphic to the group of all global sections of a field $(G, p, T)$ over the same base space and its stalks are the group of star automorphisms of $M_{2}(C)$ it $t \neq \omega$ and the group with two elements if $t=\omega$. Every section of $(G, p, T)$ is given by a sequence $\left(U_{k}\right)_{k}$ of $2 \times 2$ unitary matrices converging to $\left(\begin{array}{ll}1 & 0 \\ 0 & 1\end{array}\right)$ or to $\left(\begin{array}{ll}0 & 1 \\ 1 & 0\end{array}\right)$ in 
the strong operator topology on the set of diagonal matrices. Indeed, to a continuous section of $(G, p, T)$ there is associated a sequence $\left(U_{k}\right)_{k}$ of unitary $2 \times 2$ matrices such that if $X=\left(X_{k}\right)_{k}$ belongs to $A$ and $X_{\omega}$ is its limit, then $\lim U_{k} X_{k} U_{k}^{*}=\varphi\left(X_{\omega}\right)$ where $\varphi$ is a star automorphism of $C^{2}$.

But $\lim U_{k} X_{k} U_{k}^{*}=\lim U_{k} X_{\omega} U_{k}^{*}$, thus $U_{k} D U_{k}$ converges to $D$ or to $\left(\begin{array}{ll}d_{2} & 0 \\ 0 & d_{1}\end{array}\right)$ for every diagonal matrix $D=\left(\begin{array}{ll}d_{1} & 0 \\ 0 & d_{2}\end{array}\right)$. The sequence $\left(U_{k}\right)_{k}$ is uniquely determined up to a a sequence $\left(D_{k}\right)_{k}, D_{k}=\left(\begin{array}{ll}d_{k} & 0 \\ 0 & d_{k}\end{array}\right)$ with $\left|d_{k}\right|=1$.

Now let $J$ be the Lie algebra of derivations of $A$. Then $J$ is isomorphic to the Lie algebra of all global sections of the field $(L, q, T)$ obtained from $(E, \pi, T)$.

Every derivation of $M_{2}(C)$ is inner and $C^{2}$ being commutative has no nonzero derivations. Then every global section of $(L, q, T)$ is given by a sequence $\left(V_{k}\right)_{k}$ of complex $2 \times 2$ matrices converging to zero in the strong operator topology on the set of diagonal matrices, i.e. $V_{k} D-D V_{k}$ converges to zero for every diagonal matrix $D$.

\section{REFERENCES}

1. J. Dauns and K. H. Hofmann, The representation of biregular rings by sheaves, Math. Z., 91 (1966), 103-123.

2. - Representation of rings by sections, Mem. Amer. Math. Soc., 83 (1968).

3. H. Halpern, Irreducible module homomorphisms of a von Neumann algebra into its center, Trans. Amer. Math. Soc., 140 (1969), 194-221.

4. K. H. Hofmann, Representation of algebras by continuous sections, Bull. Amer. Math. Soc., 78 (1972), 291-373.

5. J. R. Ringrose, Cohomology of Operator Algebras, Lectures Notes in Math. 247, Springer-Verlag, Berlin and New York 1971.

6. S. Teleman, Theory of Harmonic Algebras with Application to von Neumann Algebras and the Cohomology of Locally Compact Spaces (De Rham's theorem), Lectures Notes in Math. 248, Springer-Verlag, Berlin and New York, 1971.

7. J. Tomiyama and M. Takesaki, Applications of fiber bundles to the certain class

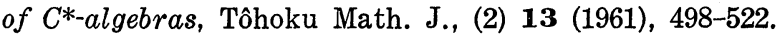

8. J. Varela, Duality of $C^{*}$-algebras. Recent advances in the representation theory of rings and $C^{*}$ algebras by continuous sections, Mem. Amer. Math. Soc., Number 148 (1974) $97-108$.

9. - Sectional representation of Banach modules, Math. Z., 139 (1974), 55-71.

Received October 9, 1974 and in revised form March 25, 1975. Research with partial support of COLCIENCIAS.

Universidad Nacional de Colombia

Current address: Apartado aéreo 19802

Bogotá, Colombia, S. A. 


\section{PACIFIC JOURNAL OF MATHEMATICS}

\section{EDITORS}

RICHARD ARENS (Managing Editor) University of California

Los Angeles, California 90024

\section{R. A. Beaumont}

University of Washington Seattle, Washington 98105
J. DugundJI Department of Mathematics University of Southern Californıa Los Angeles, California 90007

D. Gilbarg aNd J. Milgram Stanford University Stanford, California 94305

\section{ASSOCIATE EDITORS}

E. F. BeCKENBACH

B. H. NeUmanN

F. Wolf

K. YoSHIDA

\section{SUPPORTING INSTITUTIONS}

UNIVERSITY OF BRITISH COLUMBIA CALIFORNIA INSTITUTE OF TECHNOLOGY UNIVERSITY OF CALIFORNIA MONTANA STATE UNIVERSITY UNIVERSITY OF NEVADA NEW MEXICO STATE UNIVERSITY OREGON STATE UNIVERSITY UNIVERSITY OF OREGON OSAKA UNIVERSITY
UNIVERSITY OF SOUTHERN CALIFORNIA STANFORD UNIVERSITY UNIVERSITY OF TOKYO UNIVERSITY OF UTAH WASHINGTON STATE UNIVERSITY UNIVERSITY OF WASHINGTON AMERICAN MATHEMATICAL SOCIETY NAVAL WEAPONS CENTER 


\section{Pacific Journal of Mathematics \\ Vol. 59, No. 1 \\ May, 1975}

Shashi Prabha Arya and M. K. Singal, More sum theorems for topological

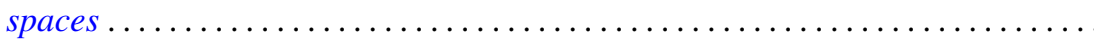

Goro Azumaya, F. Mbuntum and Kalathoor Varadarajan, On M-projective and

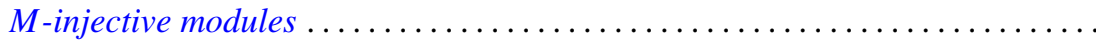

Kong Ming Chong, Spectral inequalities involving the infima and suprema of

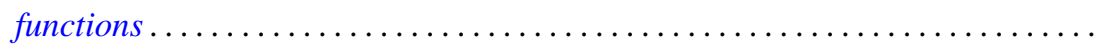

Alan Hetherington Durfee, The characteristic polynomial of the monodromy .......

Emilio Gagliardo and Clifford Alfons Kottman, Fixed points for orientation

preserving homeomorphisms of the plane which interchange two points ......

Raymond F. Gittings, Finite-to-one open maps of generalized metric spaces .......

Andrew M. W. Glass, W. Charles (Wilbur) Holland Jr. and Stephen H. McCleary,

$a^{*}$-closures of completely distributive lattice-ordered groups .............

Matthew Gould, Endomorphism and automorphism structure of direct squares of

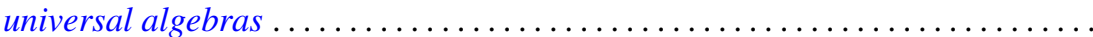

R. E. Harrell and Les Andrew Karlovitz, On tree structures in Banach spaces .....

Julien O. Hennefeld, Finding a maximal subalgebra on which the two Arens

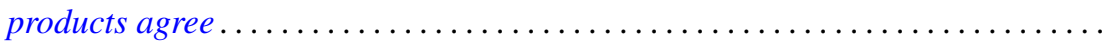

William Francis Keigher, Adjunctions and comonads in differential algebra .......

Robert Bernard Kelman, A Dirichlet-Jordan theorem for dual trigonometric

series

Allan Morton Krall, Stieltjes differential-boundary operators. III. Multivalued operators-linear relations...

Hui-Hsiung Kuo, On Gross differentiation on Banach spaces .

Tom Louton, A theorem on simultaneous observability ...

Kenneth Mandelberg, Amitsur cohomology for certain extensions of rings of algebraic integers.

Coy Lewis May, Automorphisms of compact Klein surfaces with boundary . . .

Peter A. McCoy, Generalized axisymmetric elliptic functions .

211

Muril Lynn Robertson, Concerning Siu's method for solving $y^{\prime}(t)=F(t$, $y(g(t)))$. .

Richard Lewis Roth, On restricting irreducible characters to normal

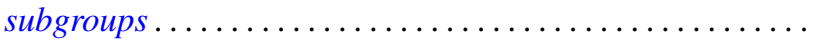

Albert Oscar Shar, $P$-primary decomposition of maps into an $H$-space .

Kenneth Barry Stolarsky, The sum of the distances to certain pointsets on the unit circle.

Bert Alan Taylor, Components of zero sets of analytic functions in $C^{2}$ in the unit ball or polydisc

Michel Valadier, Convex integrands on Souslin locally convex spaces ...

Januario Varela, Fields of automorphisms and derivations of $C$

Arnold Lewis Villone, A class of symmetric differential operators with deficiency

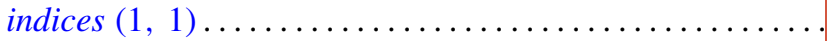

